\title{
Synchronization Conditions for Multi-agent Systems with Intrinsic Nonlinear Dynamics
}

\author{
Dongkun Han* and Graziano Chesi
}

\begin{abstract}
This paper studies local and global synchronization in multi-agent systems with nonlinear dynamics with respect to equilibrium points and periodic orbits. For local synchronization, a method is proposed based on the transformation of the original system into an uncertain polytopic system and on the use of homogeneous polynomial Lyapunov functions (HPLFs). For global synchronization, another method is proposed based on the search for a suitable polynomial Lyapunov function (PLF). The proposed methods exploit linear matrix inequalities (LMIs) and have several advantages. In particular, the proposed methods require the solution of convex optimization problems. Also, the proposed methods exploit more complex Lyapunov functions than the quadratic Lyapunov functions (QLFs) typically considered in the literature and included in this paper as a special case.
\end{abstract}

\section{INTRODUCTION}

Synchronization of complex networks has been attracting a considerable amount of academic interest, due to its broad applications in various scientific communities such as world wide web, neural networks, wireless communication and electrical power grid. Another hot topic is consensus in multi-agent systems, which shares common features with synchronization, see for instance [1]-[6].

Lyapunov methods have been successfully applied to derive synchronization conditions. In particular, a synchronization problem is investigated in [4] by using edge-based adaptation laws via a Lyapunov theoretic approach. In [7], both local and global synchronization in complex networks are investigated through generalized algebraic connectivity. In [8], some results are proposed under the assumption that the network is weighted balanced, and a distributed algorithm is proposed via non-smooth analysis.

In this paper, local and global synchronization problems of multi-agent systems with nonlinear dynamics are studied. For local synchronization, a method is proposed based on the transformation of the original system into an uncertain polytopic system and on the use of HPLFs. For global synchronization, another method is proposed based on the search for a suitable PLF. The proposed methods exploit LMIs and have several advantages. In particular, the proposed methods require the solution of convex optimization problems. Also, the proposed methods exploit more complex Lyapunov functions than the QLFs typically considered in the literature and included in this paper as a special case.

Manuscript received June 2, 2012, revised September 15, 2012 and November 19, 2012. Accepted for publication December 26, 2012.

Copyright (c) 2012 IEEE. Personal use of this material is permitted. However, permission to use this material for any other purposes must be obtained from the IEEE by sending an email to pubs-permissions@ieee.org

All authors are with the Department of Electrical and Electronic Engineering, The University of Hong Kong, Pokfulam Road, Hong Kong. E-mail: dkhan@eee.hku.hk

\section{Preliminaries}

\section{A. Problem Formulation}

The notation used throughout the paper is as follows: $\mathbb{N}, \mathbb{R}$ : natural and real number sets; $\mathbb{R}_{0}^{n}: \mathbb{R}^{n} \backslash\left\{0_{n}\right\} ; A^{\prime}$ : transpose of $A ; A>0(A \geq 0)$ : symmetric positive definite (semidefinite) matrix $A ; 0_{n}$ : origin of $\mathbb{R}^{n} ; I_{n}: n \times n$ identity matrix; $A \otimes$ $B$ : Kronecker product of matrices $A$ and $B$; he $(A): A+A^{\prime}$, with $A \in \mathbb{R}^{n \times n} ; \operatorname{co}\left\{X_{1}, \ldots, X_{p}\right\}$ : convex hull of matrices $X_{1}, \ldots, X_{p} \in \mathbb{R}^{m \times n} ; X^{[i]}: i$-th Kronecker power, i.e.

$$
X^{[i]}= \begin{cases}X \otimes X^{[i-1]} & \text { if } i>1 \\ 1 & \text { if } i=0 .\end{cases}
$$

Let $\mathscr{G}=(\mathscr{A}, \mathscr{E}, G)$ be a weighted and directed graph, where $\mathscr{A}=\left\{A_{1}, \ldots, A_{n}\right\}$ is a finite nonempty set to describe the set of $n$ nodes of a multi-agent system, $\mathscr{E}$ is the set of directed edges belonging to $\mathscr{A} \times \mathscr{A}$, and $G$ is a $n \times n$ weighted adjacency matrix. A directed edge from $A_{j}$ to $A_{i}$ is described by $G_{i j}$ which represents an information transmitting channel from the $j$-th node to the $i$-th node.

In this paper, we investigate multi-agent systems with directional information exchange described by

$$
\dot{x}_{i}(t)=f\left(x_{i}(t)\right)-c \sum_{j=1}^{N} L_{i j} \Gamma x_{j}(t), \quad i, j=1, \ldots, N
$$

where $x_{i} \in \mathbb{R}^{n}$ is the state of the $i$-th agent, $N$ is the number of agents, $c$ is the coupling weight, $f\left(x_{i}\right) \in \mathbb{R}^{n}$ is a nonlinear function, $\Gamma=\operatorname{diag}\left(\gamma_{1}, \ldots, \gamma_{n}\right) \in \mathbb{R}^{n \times n}$ is a diagonal matrix where $\gamma_{i}>0$ stands for an agent communicating through the $i$-th state, and $L_{i j}$ is the $i j$-th entry of the Laplacian matrix $L \in \mathbb{R}^{N \times N}$ given by $L_{i j}=-G_{i j}$ for all $i \neq j$ and by $L_{i i}=-\sum_{j=1, j \neq i}^{N} L_{i j}$.

We can rewrite the uncertain multi-agent dynamical system (1) in compact form as

$$
\dot{x}(t)=g(x(t))-c(L \otimes \Gamma) x(t)
$$

where $x(t)=\left(x_{1}(t)^{\prime}, \ldots, x_{N}(t)^{\prime}\right)^{\prime}$ and $g(x(t))=$ $\left(f\left(x_{1}(t)\right)^{\prime}, \ldots, f\left(x_{N}(t)\right)^{\prime}\right)^{\prime}$. Let $s(t) \in \mathbb{R}^{n}$ be a solution of an isolated node, i.e.

$$
\dot{s}(t)=f(s(t)) .
$$

Let us observe that $s(t)$ can be either an equilibrium point, a periodic orbit, or a chaotic orbit. Then, two synchronization problems are proposed as follows.

Problem 1: To establish if the multi-agent dynamical system (2) achieves local synchronization, i.e. for any $\epsilon$ there exist $\kappa(\epsilon)$ and $T>0$ such that $\left\|x_{i}(0)-x_{j}(0)\right\| \leq \kappa(\epsilon)$ implies $\left\|x_{i}(t)-x_{j}(t)\right\| \leq \epsilon$ for all $t>T$ and $i, j=1, \ldots, N$.

Problem 2: To establish if the multi-agent dynamical system (2) achieves global synchronization, i.e. for any $\epsilon$ there 
exist $T>0$ such that $\left\|x_{i}(t)-x_{j}(t)\right\| \leq \epsilon$ for all $t>T$ and $i, j=1, \ldots, N$ (regardless of $\left\|x_{i}(0)-x_{j}(0)\right\|$ ).

\section{B. Positive Polynomials via LMIs}

A useful tool for establishing whether a polynomial is nonnegative consists of establishing whether it is a SOS polynomial, and this is equivalent to an LMI feasibility test as explained in [9] and references therein.

Specifically, let $x \in \mathbb{R}^{r}$ and let $h(x)$ be a polynomial with all the monomials having degree between $2 l$ and $2 \mathrm{~m}$. Let $x^{\{l, m\}} \in \mathbb{R}^{\sigma(r, l, m)}$ be a vector containing all monomials of degree between $l$ and $m$ where

$$
\sigma(r, l, m)=\frac{(r+m) !}{r ! m !}-\frac{(r+l-1) !}{r !(l-1) !}
$$

Then, $h(x)$ can be written according to the square matrix representation (SMR) as

$$
h(x)=x^{\{l, m\}^{\prime}}(H+E(\delta)) x^{\{l, m\}}
$$

where $H \in \mathbb{R}^{\sigma(r, m, l) \times \sigma(r, m, l)}$ is a symmetric matrix, and $E(\delta)$ is a linear parametrization of the linear subspace

$$
\mathcal{E}=\left\{E=E^{\prime}: x^{\{l, m\}^{\prime}} E x^{\{l, m\}}=0\right\} .
$$

Observe that $E(\delta)$ can be simply generated with standard software since it is a parametrization of the solutions of a system of linear equations.

The representation (5) allows one to establish whether a polynomial is SOS via LMIs. Indeed, $h(x)$ is SOS if there exist polynomials $h_{1}(x), h_{2}(x), \ldots$ such that

$$
h(x)=\sum_{i} h_{i}(x)^{2}
$$

and this condition holds if and only if there exists $\delta$ such that the following LMI holds:

$$
H+E(\delta) \geq 0 .
$$

In the sequel of the paper we assume that $l$ and $m$ are the largest and the smallest integers, respectively, such that the monomials of $h(x)$ have degree between $2 l$ and $2 m$.

\section{CONDITIONS FOR LOCAL SYNCHRONIZATION}

\section{A. System Transformation}

For local synchronization we introduce the following assumption on $f\left(x_{i}\right)$.

Assumption 1: The function $f\left(x_{i}\right)$ is continuously differentiable in a neighborhood of the solution $s(t)$.

Remark 1: This assumption is very mild one as it just requires that the first derivative of the vector field is continuous in a neighborhood of the solution of interest.

Let us subtract (3) from (1). We get the system

$$
\dot{y}_{i}(t)=f\left(x_{i}(t)\right)-f(s(t))-c \sum_{j=1}^{N} L_{i j} \Gamma y_{j}(t)
$$

where $y_{i}=x_{i}-s, i=1, \ldots, N$. The system (9) can be linearized around $s(t)$ as

$$
\dot{y}(t)=\left(I_{N} \otimes D f(s(t))\right) y(t)-c(L \otimes \Gamma) y(t)
$$

where $y(t)=\left(y_{1}(t)^{\prime}, \ldots, y_{N}(t)^{\prime}\right)^{\prime}$ and $D f(s(t)) \in \mathbb{R}^{n \times n}$ is the Jacobian matrix of $f\left(x_{i}\right)$ evaluated for $x_{i}=s(t)$. Let us define $z_{i}=y_{1}-y_{i}, i=2, \ldots, N$, and let $z(t)=$ $\left(z_{2}(t)^{\prime}, \ldots, z_{N}(t)^{\prime}\right)^{\prime}$. We obtain a reduced system as

$$
\begin{aligned}
\dot{z}(t) & =A(t) z(t) \\
& =\left(I_{N-1} \otimes D f(s(t))-c(\widetilde{L} \otimes \Gamma)\right) z(t)
\end{aligned}
$$

where

$$
\widetilde{L}=\left(\begin{array}{ccc}
L_{22}-L_{12} & \ldots & L_{2 N}-L_{1 N} \\
\vdots & \ddots & \vdots \\
L_{N 2}-L_{12} & \ldots & L_{N N}-L_{1 N}
\end{array}\right)
$$

The next result directly follows from the definition of local synchronization.

Lemma 1: Suppose that Assumption 1 holds. The local synchronization of system (2) can be achieved if the system (11) is asymptotically stable.

The next step consists of transforming (11) into an uncertain polytopic system of the form

$$
\left\{\begin{array}{l}
\dot{z}(t)=\widehat{A}(p(t)) z(t) \\
p(t) \in \mathscr{P}
\end{array}\right.
$$

where $p(t) \in \mathbb{R}^{q}$ is an uncertain parameter vector, $\mathscr{P}$ is the simplex defined by

$$
\mathscr{P}=\operatorname{co}\left\{p^{(1)}, \ldots, p^{(w)}\right\}
$$

and $\widehat{A}(p(t))$ is given by

$$
\widehat{A}(p(t))=\widehat{A}_{0}+\sum_{i=1}^{q} p_{i}(t) \widehat{A}_{i}
$$

for some $\widehat{A}_{0}, \widehat{A}_{1}, \ldots, \widehat{A}_{q} \in \mathbb{R}^{k \times k}$. This can be done by choosing any bounds $b_{i j}, c_{i j} \in \mathbb{R}$ satisfying

$$
b_{i j} \leq A_{i j}(t) \leq c_{i j} \forall t \geq 0
$$

for all $i, j=1, \ldots, k$. Observe that such bounds exist since $D f(s(t))$ is continuous. Then, a parameter $p_{l}(t)$ is assigned to each entry of $A_{i j}(t)$ choosing

$$
\left\{\begin{array}{l}
\widehat{A}_{0, i j}=b_{i j} \\
\widehat{A}_{l, i j}=c_{i j}-b_{i j}
\end{array}\right.
$$

in order to ensure that the uncertain polytopic system includes (11). Clearly, for entries of $A_{i j}(t)$ that are linearly dependent, one can introduce one parameter $p_{l}(t)$ only.

\section{B. Local Synchronization Conditions}

Robust stability of (12) can be investigated by HPLFs, a non-conservative class of Lyapunov functions whose construction can be tackled through LMIs, see e.g. [10]. In order to derive an LMI condition based on HPLFs for local synchronization of (1), let us introduce the following result.

Theorem 1: Suppose that Assumption 1 holds. The local synchronization of (1) can be achieved if there exists a continuously differentiable homogeneous function $v(z)$ such that

$$
\forall z \neq 0 \quad\left\{\begin{array}{l}
0<v(z) \\
0<-\varrho_{i}(z) \quad \forall i=1, \ldots, w
\end{array}\right.
$$


where

$$
\varrho_{i}(z)=\left.\dot{v}(z, p)\right|_{p=p^{(i)}}
$$

and

$$
\dot{v}(z, p)=\left(\frac{d v(z)}{d z}\right)^{\prime}(\widehat{A}(p) z) .
$$

Such a $v(z)$ is a homogeneous Lyapunov function for (12). Proof Suppose that (13) holds. Let us observe that

$$
\dot{v}(z, p)=\sum_{i=1}^{w} d_{i}(p) \varrho_{i}(z)
$$

where $d_{1}(p), \ldots, d_{w}(p) \in \mathbb{R}$ are such that

$$
\left\{\begin{array}{l}
\sum_{\substack{i=1 \\
w}}^{w} d_{i}(p) p^{(i)}=p \\
\sum_{i=1}^{w} d_{i}(p)=1 \\
d_{i}(p) \geq 0 \quad \forall i=1, \ldots, w .
\end{array}\right.
$$

Hence, (13) implies that

$$
\dot{v}(z, p)<0 \quad \forall z \neq 0
$$

i.e. $v(z)$ is a Lyapunov function for (12) for all $p \in \mathscr{P}$, in particular a homogeneous Lyapunov function. Therefore, (12) is robustly asymptotically stable, and local synchronization of (1) can be achieved.

Let $v(z)$ be a homogeneous polynomial of degree $2 m$. We can express $v(z)$ via the SMR as

$$
v(z)=z^{\{m, m\}^{\prime}} V z^{\{m, m\}}
$$

where $V \in \mathbb{R}^{\sigma((N-1) n, m, m) \times \sigma((N-1) n, m, m)}$ is a symmetric matrix. In order to derive the LMI condition for local synchronization, let us introduce the following definition.

Definition 1: Let $\widehat{A}^{\#}$ be the matrix satisfying

$$
\frac{d z^{\{m, m\}}}{d t}=\frac{\partial z^{\{m, m\}}}{\partial z} \widehat{A} z=\widehat{A}^{\#} z^{\{m, m\}} .
$$

Then, $\widehat{A}^{\#}$ is called extended matrix of $\widehat{A}$.

Lemma 2: [11] Let $z^{[m]}$ be the $m$-th Kronecker power of $z$, and $K_{m}$ be the matrix satisfying $z^{[m]}=K_{m} z^{\{m, m\}}$. Then,

$$
\widehat{A}^{\#}=\left(K_{m}^{\prime} K_{m}\right)^{-1} K_{m}^{\prime}\left(\sum_{i=0}^{m-1} I_{m-1-i} \otimes \widehat{A} \otimes I_{i}\right) K_{m} .
$$

Let us define

$$
\widetilde{A}_{i}=\widehat{A}\left(p^{(i)}\right)
$$

and let $\widetilde{A}_{i}^{\#}$ be the extended matrix of $\widetilde{A}_{i}$. The LMI condition for local synchronization is obtained as follows.

Theorem 2: Suppose that Assumption 1 holds. For any $m \geq 1$, let $E(\delta)$ be a linear parametrization of the linear subspace (6) with $l=m$ (see Section II-B for details). The local synchronization of (1) can be achieved if there exist a symmetric matrix $V$ and $\delta^{(1)}, \ldots, \delta^{(w)}$ such that

$$
\left\{\begin{array}{l}
0<V \\
0<- \text { he }\left(V \widetilde{A}_{i}^{\#}\right)-E\left(\delta^{(i)}\right) \quad \forall i=1, \ldots, w .
\end{array}\right.
$$

Proof Suppose that (15) holds. Pre- and post-multiplying the first LMI in (15) by $z^{\{m, m\}^{\prime}}$ and $z^{\{m, m\}}$, respectively, one has that

$$
\begin{aligned}
0 & <z^{\{m, m\}^{\prime}} V z^{\{m, m\}} \\
& =v(z)
\end{aligned}
$$

hence implying that $v(z)$ is positive definite since $z^{\{m, m\}^{\prime}} z^{\{m, m\}}>0$ for all $z \neq 0$. From (14) it follows that

$$
\varrho_{i}(z)=z^{\{m, m\}^{\prime}} h e\left(V \widetilde{A}_{i}^{\#}\right) z^{\{m, m\}}
$$

and hence from the second LMI one has that $\varrho_{i}(z)$ is negative definite. Hence, from Theorem 1 it follows that $v(z)$ is a HPLF for (12), and therefore the local synchronization of (1) can be achieved.

Let us observe that one can systematically establish if there exist a symmetric matrix $V$ and $\delta^{(1)}, \ldots, \delta^{(w)}$ such that (15) holds. In fact, this is an LMI condition, which amounts to solving a convex optimization problem, see [9] and references therein for details.

\section{CONDITIONS FOR GLOBAL SYNCHRONIZATION}

In order to investigate the global synchronization of (1), let us rewrite (9) as

$$
\dot{y}(t)=\psi(y(t), s(t))-c(L \otimes \Gamma) y(t)
$$

where $y(t)=\left(y_{1}(t)^{\prime}, \ldots, y_{N}(t)^{\prime}\right)^{\prime}, \quad \psi(y(t), s(t))=$ $\left(\psi\left(y_{1}(t), s(t)\right)^{\prime}, \ldots, \psi\left(y_{N}(t), s(t)\right)^{\prime}\right)^{\prime}$ and

$$
\psi\left(y_{i}(t), s(t)\right)=f\left(y_{i}(t)+s(t)\right)-f(s(t)), \quad i=1, \ldots, N .
$$

Let us introduce the following assumption on $f(x)$.

Assumption 2: The function $f\left(x_{i}\right)$ is polynomial.

Remark 2: Various existing approaches for global synchronization like [4], [6], [7] assume the QUAD condition (or oneside Lipschits condition). However, the QUAD condition is not satisfied for simple nonlinearities such as quadratic and cubic functions. Instead, Assumption 2 includes such nonlinearities, and also includes important systems such as Lorenz system and Hamiltonian systems. Moreover, continuous functions can be approximated arbitrarily well by polynomial ones, which means that Assumption 2 is indeed mild.

The following result directly follows from [12].

Lemma 3: Let $\sigma=\left(\sigma_{1}, \ldots, \sigma_{N}\right)^{\prime}$ with $\sigma_{i}>0, i=$ $1, \ldots, N$, and $\sum_{i=1}^{N} \sigma_{i}=1$. The global synchronization of (1) can be achieved if there exists a matrix

$$
M=\left(I_{N}-1_{N} \sigma^{\prime}\right) \otimes I_{n}
$$

such that

$$
\lim _{t \rightarrow \infty}\|M y(t)\|=0
$$

For ease of description, let us consider first the case where $s(t)$ is constant. We have the following result.

Theorem 3: Suppose that Assumption 2 holds. The global synchronization of (1) can be achieved if there exist $\varepsilon \in \mathbb{R}$, 
a continuously differentiable function $v(y)$, and two functions $u_{1}(y)$ and $u_{2}(y)$ such that

$$
\left\{\begin{aligned}
0 & \leq \varphi_{i}(y) \quad \forall y \quad \forall i=1, \ldots, 4 \\
0 & <\varepsilon
\end{aligned}\right.
$$

where

$$
\begin{aligned}
& \varphi_{1}(y)=u_{1}(y)-\varepsilon \\
& \varphi_{2}(y)=u_{2}(y)-\varepsilon \\
& \varphi_{3}(y)=v(y)-u_{1}(y)\|M y\|^{2} \\
& \varphi_{4}(y)=-\dot{v}(y)-u_{2}(y)\|M y\|^{2}
\end{aligned}
$$

and

$$
\dot{v}(y)=\left(\frac{d v(y)}{d y}\right)^{\prime}(\psi(y, s)-c(L \otimes \Gamma) y) .
$$

Proof Suppose that (20) holds. From the first inequality for $i=3$ we get

$$
v(y) \geq u_{1}(y)\|M y\|^{2}
$$

and, since $u_{1}(y)$ is positive from the first inequality for $i=1$,

$$
v(y)>0 \quad \forall y: M y \neq 0 .
$$

Similarly, for $i=4$ we obtain that

$$
\dot{v}(y)<0 \quad \forall y: M y \neq 0 .
$$

Hence, $v(y)$ is positive and its time derivative is negative whenever $M y \neq 0$. This implies that (19) holds, and therefore global synchronization of (1) can be achieved.

Theorem 3 provides a condition for global synchronization of (1) based on the idea of searching for a Lyapunov function $v(y)$ proving (19). Let us observe that the role of the term $M y$ in the definition of $\varphi_{3}(y)$ and $\varphi_{4}(y)$ is to require that $v(y)$ and $-\dot{v}(y)$ are positive whenever synchronization is not achieved, since this implies that $v(y)$ will decrease till $M y$ vanishes.

In order to check the condition of Theorem 3 via LMIs, we consider the case where $v(y), u_{1}(y)$ and $u_{2}(y)$ are polynomials. Clearly, $v(y)$ has no constant and linear monomials if it has to satisfy (20). Hence, let us parametrize $v(y), u_{1}(y)$ and $u_{2}(y)$ as

$$
\begin{aligned}
v(y) & =w_{0}^{\prime} y^{\left\{2,2 m_{0}\right\}} \\
u_{i}(y) & =w_{i}^{\prime} y^{\left\{0,2 m_{i}\right\}}, \quad i=1,2
\end{aligned}
$$

where, for all $i=0,1,2, m_{i}$ is an integer and $w_{i}$ is a vector of suitable size. Let us express $\varphi_{i}(y), i=1, \ldots, 4$ via the SMR as

$$
\varphi_{i}(y)=y^{\left\{l_{i}, m_{i}\right\}^{\prime}}\left(\Phi_{i}(\varepsilon, w)+E_{i}\left(\delta_{i}\right)\right) y^{\left\{l_{i}, m_{i}\right\}}
$$

where $w=\left(w_{0}^{\prime}, w_{1}^{\prime}, w_{2}^{\prime}\right)^{\prime}$.

Theorem 4: Suppose that Assumption 2 holds. The global synchronization of (1) can be achieved if there exist $\varepsilon, w$ and $\delta_{i}, i=1, \ldots, 4$, such that

$$
\left\{\begin{aligned}
0 & \leq \Phi_{i}(\varepsilon, w)+E_{i}\left(\delta_{i}\right) \quad \forall i=1, \ldots, 4 \\
0 & <\varepsilon
\end{aligned}\right.
$$

Proof Suppose that (25) holds. Pre- and post-multiplying the first LMI in (25) by $y^{\left\{l_{i}, m_{i}\right\}^{\prime}}$ and $y^{\left\{l_{i}, m_{i}\right\}}$, respectively, one gets

$$
\begin{aligned}
0 & \leq y^{\left\{l_{i}, m_{i}\right\}^{\prime}}\left(\Phi_{i}(\varepsilon, w)+E_{i}\left(\delta_{i}\right)\right) y^{\left\{l_{i}, m_{i}\right\}} \\
& =\varphi_{i}(y) \quad \forall y \forall i=1, \ldots, 4 .
\end{aligned}
$$

Consequently, (20) holds, and from Theorem 3 we conclude that the global synchronization of (1) can be achieved.

Theorem 4 provides the sought LMI condition for global synchronization of (1). This condition can be directly extended to the case where $s(t)$ is either a periodic orbit or a chaotic orbit by introducing an uncertain polytopic system as done in Section III-A and by repeating the LMI condition in Theorem 4 at the vertices of the polytope. The details are omitted for conciseness.

\section{NUMERICAL EXAMPLES}

In this section, two examples are provided to illustrate the proposed approach. These examples are deliberately simple for ease of description and due to space limits. The computations are done in MATLAB with the toolbox SeDuMi [13].

\section{A. Example for Local Synchronization}

Let us consider a two-agent system where each agent has a second order dynamics. The model (1) is described by a nonlinear function $f(x)$ given by

$$
f\left(x_{i}\right)=\left(\begin{array}{c}
x_{i 1}-x_{i 2}-x_{i 1}\left(x_{i 1}^{2}+x_{i 2}^{2}\right) \\
x_{i 1}+x_{i 2}-x_{i 2}\left(x_{i 1}^{2}+x_{i 2}^{2}\right)
\end{array}\right)
$$

where $x_{i}=\left(x_{i 1}, x_{i 2}\right)^{\prime}, i=1,2$. The linear part of (1) is described by the constants

$$
c=1, \quad \Gamma=I_{2}, \quad G=\left(\begin{array}{ll}
1 & 1 \\
2 & 1
\end{array}\right) .
$$

We have that (3) holds with

$$
s(t)=\left(\begin{array}{l}
0 \\
0
\end{array}\right) \text { or } s(t)=\left(\begin{array}{c}
\cos t \\
\sin t
\end{array}\right) .
$$

Let us consider the problem of establishing local consensus for the second solution of $s(t)$, i.e. for the periodic orbit. The matrix $A(t)$ in (11) is given by

$$
A(t)=\left(\begin{array}{cc}
-2-3 \cos ^{2} t-\sin ^{2} t & -1-2 \cos t \sin t \\
1-2 \cos t \sin t & -2-\cos ^{2} t-3 \sin ^{2} t
\end{array}\right) .
$$

As described in Section III-A, one can embed (11) into an uncertain polytopic system. In particular, by choosing $p_{1}=$ $\cos ^{2} t$ and $p_{2}=\cos t \sin t$, it follows that $\widehat{A}(p)$ in (12) is given by

$$
\widehat{A}(p)=\left(\begin{array}{cc}
-3-2 p_{1} & -1-2 p_{2} \\
1-2 p_{2} & -5+2 p_{1}
\end{array}\right) .
$$

Let us observe that $p_{1} \in[0,1], p_{2} \in[-0.5,0.5]$, and hence the polytope $\mathscr{P}$ is given by

$$
\mathscr{P}=\operatorname{co}\left\{\left(\begin{array}{c}
1 \\
0.5
\end{array}\right),\left(\begin{array}{c}
1 \\
-0.5
\end{array}\right),\left(\begin{array}{c}
0 \\
0.5
\end{array}\right),\left(\begin{array}{c}
0 \\
-0.5
\end{array}\right)\right\} .
$$


We find that the LMI condition (15) holds and hence local synchronization can be achieved according to Theorem 2 . In particular, a HPLF for this case is given by $v(z)=z_{21}^{4}+$ $z_{21}^{2} z_{22}^{2}+z_{22}^{4}$.

For completeness, we report in Figure 1 some simulations. In particular, the first subfigure shows the trajectory of $x(t)$ for the initial condition $x(0)=(1,2,-1,-2)^{\prime}$, while the second subfigure shows 100 trajectories for $z(t)$ with initial conditions randomly chosen in $[-10,10]^{4}$.
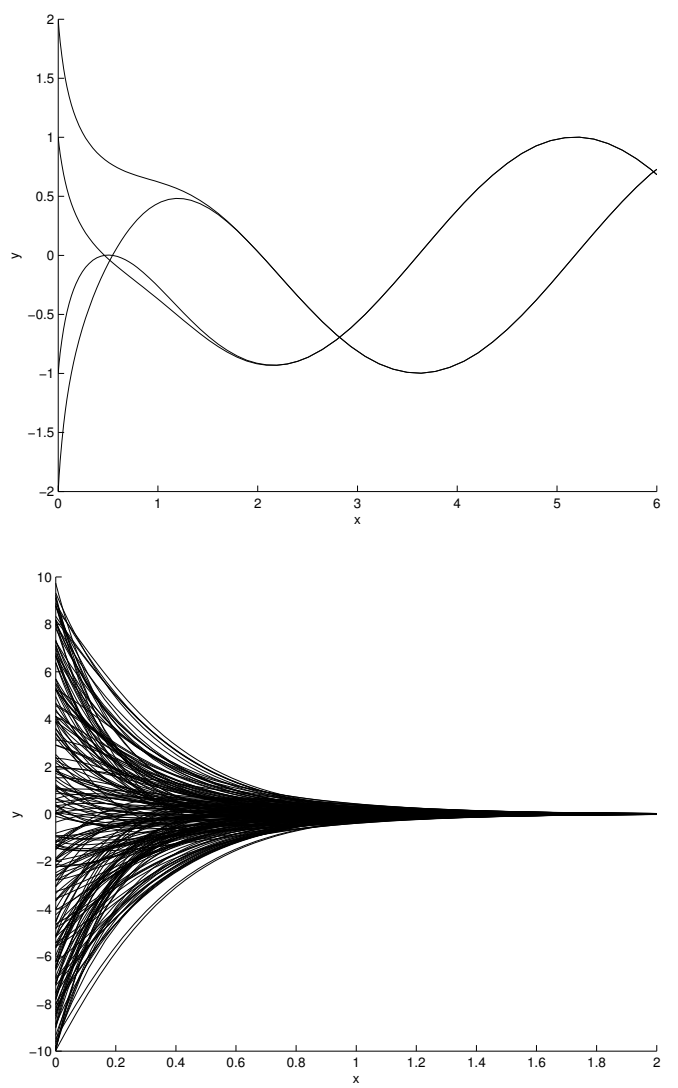

Fig. 1. Example for local synchronization.

\section{B. Example for Global Synchronization}

Let us consider (1) with

$$
f\left(x_{i}\right)=\left(\begin{array}{c}
-x_{i 2} \\
-x_{i 1}-x_{i 1}^{3}-x_{i 2}
\end{array}\right)
$$

where $x_{i}=\left(x_{i 1}, x_{i 2}\right)^{\prime}, i=1,2$, and

$c=1, \Gamma=I_{2}, \quad G=\left(\begin{array}{ll}1 & 2 \\ 1 & 1\end{array}\right), \quad M=\left(\begin{array}{cc}0.5 & -0.5 \\ -0.5 & 0.5\end{array}\right)$.

We have that (3) holds with $s(t)=(0,0)^{\prime}$. We consider the problem of establishing global consensus for this solution. To this end, we check the LMI condition (25) with auxiliary polynomials $u_{i}(y)$ of degree 2 . We find that this condition cannot be satisfied using quadratic Lyapunov functions $v(y)$. Instead, the condition is feasible with Lyapunov functions of degree 4 , in particular the condition holds with $\varepsilon=0.5$, $u_{i}(y)=1+y_{i}^{\prime} y_{i}$ and $v(y)=y_{1}^{\prime} y_{1}+y_{2}^{\prime} y_{2}+\left(y_{1}^{\prime} y_{1}\right)^{2}+$ $\left(y_{2}^{\prime} y_{2}\right)^{2}-y_{11}^{2} y_{21}^{2}-y_{12}^{2} y_{22}^{2}$. Hence, from Theorem 4, global synchronization can be achieved.

\section{CONCLUSIONS}

We have investigated local and global synchronization in multi-agent systems with nonlinear dynamics. For local synchronization, a method has been proposed based on the transformation into an uncertain polytopic system and on the use of HPLFs, while, for global synchronization, another method has been proposed based on the search for a suitable PLF. Future work can consider the extension of these methods to switching systems following the frameworks introduced in [14]-[17].

\section{REFERENCES}

[1] Z. Li and G. Chen. Global synchronization and asymptotic stability of complex dynamical networks. IEEE Transactions on Circuits and Systems-II, 53(1):28-33, 2006.

[2] Z. Li, Z. Duan, G. Chen, and L. Huang. Consensus of multi-agent systems and synchronization of complex networks: A unified viewpoint. IEEE Transactions on Circuits and Systems-I, 57(1):213-224, 2010.

[3] X. Wang and G. Chen. Synchronization in scale-free dynamical networks: Robustness and fragility. IEEE Transactions on Circuits and Systems-I, 49(1):54-62, 2002.

[4] W. Yu, P. DeLellis, G. Chen, M. D. Bernardo, and J. Kurths. Distributed adaptive control of synchronization in complex networks. IEEE Transactions on Automatic Control, 57(8):2153-2158, 2012.

[5] W. Lu. Global synchronization of discrete-time dynamical network with a directed graph. IEEE Transactions on Circuits and Systems-II, 54(2):136-140, 2007.

[6] P. DeLellis, M. D. Bernardo, and G. Russo. On QUAD, Lipschitz and contracting vector fields for consensus and synchronization of network. IEEE Transactions on Circuits and Systems-I, 58(3):576-583, 2011.

[7] W. Yu, G. Chen, and M. Cao. Consensus in directed network of agents with nonlinear dynamics. IEEE Transactions on Automatic Control, 56(6):1436-1441, 2011.

[8] J. Cortes. Distributed algorithms for reaching consensus on general functions. Automatica, 44(3):726-737, 2007.

[9] G. Chesi. LMI techniques for optimization over polynomials in control: a survey. IEEE Transactions on Automatic Control, 55(11):2500-2510, 2010 .

[10] G. Chesi. LMI conditions for time-varying uncertain systems can be non-conservative. Automatica, 47(3):621-624, 2011.

[11] G. Chesi, A. Garulli, A. Tesi, and A. Vicino. Homogeneous Polynomial Forms for Robustness Analysis of Uncertain Systems. Springer-Verlag, 2009.

[12] W. Yu, J. Cao, and J. Lü. Global synchronization of linearly hybrid coupled networks with time-varying delay. SIAM Journal on Applied Dynamic systems, 7(1):108-133, 2008.

[13] J. Sturm. Using SeDuMi 1.02, a MATLAB toolbox for optimization over symmetric cones. Mathematical Optimization Software, 11-12:625-653, 1999.

[14] L. Zhang and J. Lam. Necessary and sufficient conditions for analysis and synthesis of Markov jump linear systems with incomplete transition descriptions. IEEE Transactions on Automatic Control, 55(7):16951701, 2010.

[15] L. Zhang and P. Shi. Stability, $l_{2}$-gain and asynchronous $\mathrm{H}_{\infty}$ control of discrete-time switched systems with average dwell time. IEEE Transactions on Automatic Control, 54(9):2193-2200, 2009.

[16] L. Zhang, N. Cui, M. Liu, and Y. Zhao. Asynchronous filtering of discrete-time switched linear systems with average dwell time. IEEE Transactions on Circuits and Systems-I, 58(5):1109-1118, 2011.

[17] G. Chesi, P. Colaneri, J. C. Geromel, R. H. Middleton, and R. Shorten. A nonconservative LMI condition for stability of switched systems with guaranteed dwell time. IEEE Transactions on Automatic Control, 57(5):1297-1302, 2012. 